\title{
Bone scintigraphy for osteonecrosis of the knee in patients with non-traumatic osteonecrosis of the femoral head: comparison with magnetic resonance imaging
}

\author{
T Sakai, N Sugano, T Nishii, K Haraguchi, H Yoshikawa, K Ohzono
}

\begin{abstract}
Objective-To determine whether technetium bone scintigraphy (BS) is useful for screening of non-traumatic osteonecrosis of the knee (ONK), which was a major affected site, secondary to the femoral head, among multiple osteonecrosis, in patients with non-traumatic osteonecrosis of the femoral head (ONFH).

Methods-A total of 214 knee joints in 107 patients with ONFH were evaluated by BS and a comparison made with magnetic resonance imaging (MRI). ONK was classified into five sites, including the femoral condyles (ONFC), distal femoral metaphysis (ONFM), tibial plateau (ONTP), proximal tibial metaphysis (ONTM), and patella (ONP).
\end{abstract}

Results-Based on the diagnosis by MRI, ONK was detected in 103 knees of 62 patients $(48 \%)$. ONFC was most common (86 knees, 40\%), ONFM (15\%), followed by ONTM (10\%), ONP (3\%), and ONTP $(0.9 \%)$. Sensitivity, specificity, and accuracy of BS for ONFC detection were $63 \%$, $71 \%$, and $68 \%$, respectively. When the ONFC lesions on the coronal views of MRI were large or medium sized and occupied two thirds, or the entire anteroposterior joint surface on the sagittal views, the sensitivity of BS for ONFC detection increased to $89 \%(34 / 38$ knees $)$. The sensitivity of BS for ONFM, ONTM, and ONP detection was $3 \%, 0 \%$, and $0 \%$, respectively, but these lesions showed a low likelihood of collapse.

Conclusion-BS is useful for screening large ONK in patients with ONFH given that $89 \%$ of patients with ONFC who had a high risk of collapse of the knee were identified.

(Ann Rheum Dis 2001;60:14-20)

Non-traumatic osteonecrosis of the femoral head $(\mathrm{ONFH})$ is often accompanied by other sites of osteonecrosis as multifocal osteonecrosis ${ }^{12}$ or multiple osteonecrosis. LaPorte et al reported that when multifocal osteonecrosis was defined as a disease affecting three or more separate anatomical sites, it was found in 32/1056 (3\%) patients with osteonecrosis. ${ }^{1}$ All 32 patients had ONFH and knee involvement. They also reported that osteonecrosis was seen both in the shoulder (28 patients) and ankle (eight patients). ${ }^{1}$ In a multicentre study, using the same definition of multifocal osteonecrosis, ${ }^{2} 101$ patients with femoral head disease also had osteonecrosis of the knee $(96 \%)$, shoulder $(80 \%)$, and ankle (44\%). On the other hand, Shimizu et al reported that multiple osteonecrosis, which affected two or more separate anatomical sites, was observed by magnetic resonance imaging (MRI) screening in 167 of 250 patients $(67 \%)$ with steroid related $\mathrm{ONFH}$, and that the most common site besides the femoral head was the lateral femoral condyle (49\%), followed by distal femoral metaphysis (37\%), medial femoral condyle $(32 \%)$, and humeral head $(24 \%)$ (Steroidinduced multiple bone necroses: an analysis of 2000 joints in 250 patients. Paper presented at the Annual Meeting of the American Academy of Orthopaedic Surgeons, Anaheim, California, February, 1999). Thus the knee is a major affected site, secondary to the femoral head, among patients with multifocal or multiple osteonecrosis.

Osteonecrosis is thought to develop after an ischaemic event in bone and bone marrow. ${ }^{3}$ Although the cause of ischaemia remains unknown, Jones claimed that intravascular coagulopathy was only an intermediary event initiated by several seemingly unrelated risk factors, including alcoholism, hypercortisonism (hyperlipidaemia), and allograft organ rejection (hypersensitivity reactions). ${ }^{4}$ Intravascular coagulopathy occurs in capillarysinusoidal beds, arteries, or veins. The blood supply in the knee joint is derived from a rich anastomosis of the five major constant arteries-namely, the superior medial and lateral, the middle, and the inferior medial and lateral genicular arteries. ${ }^{5}$ According to a hypothesis based on intravascular coagulopathy, non-traumatic osteonecrosis of the knee (ONK) can affect each site of the knee: the femoral condyles (ONFC), distal femoral metaphysis (ONFM), tibial plateau (ONTP), proximal tibial metaphysis (ONTM), and patella (ONP) (fig 1). In particular, ONFC, which develops at the subchondral bone of the tibiofemoral joint, sometimes becomes symptomatic owing to collapse of the necrotic lesions $^{6-10}$ and requires conservative or operative treatment. ${ }^{1112}$

For screening of multifocal osteonecrosis, technetium bone scintigraphy (BS) has been used $^{1314}$ in patients with ONFH. BS has several advantages: many joints can be examined at one time on total body images to determine whether multiple osteonecrosis develops; and
Accepted for publication 18 May 2000 


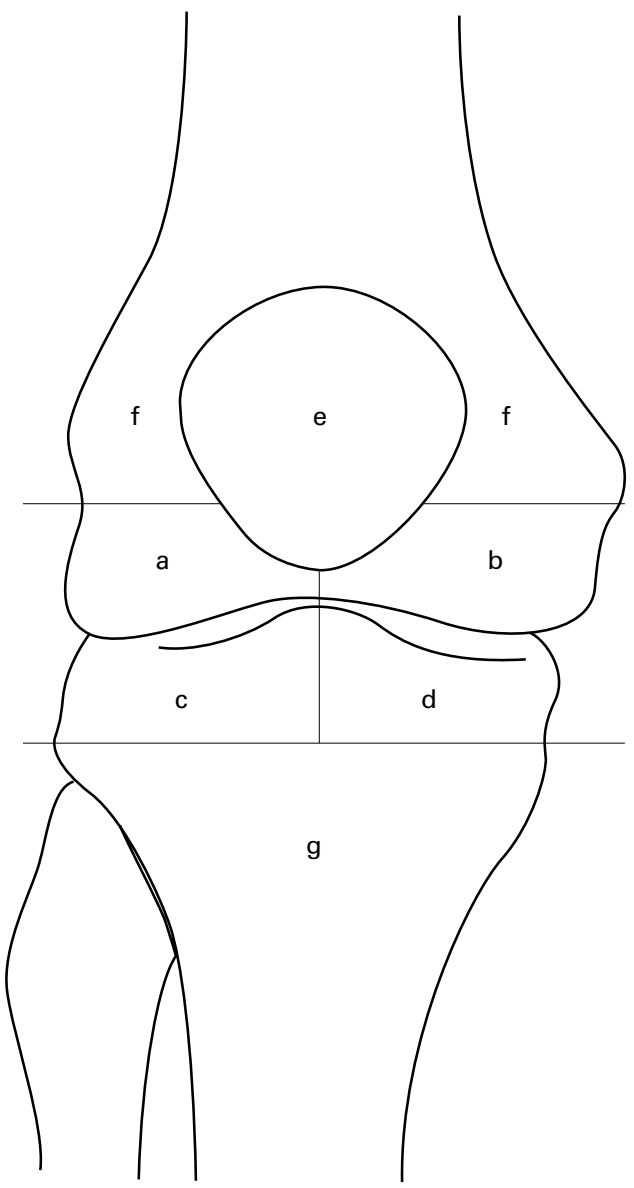

Figure 1 Seven areas of the knee (right knee). a: Lateral femoral condyle; $b$ : medial femoral condyle ( $a, b$ : ONFC); c: lateral tibial plateau; $d$ : medial tibial plateau $(c, d$ : ONTP); e: patella (ONP); f: distal femoral metaphysis (ONFM); g: proximal tibial metaphysis (ONTM).

BS can be used for patients with cardiac pacemakers, intracranial clips, and claustrophobia, who cannot undergo magnetic resonance imaging (MRI). However, BS of the hip has been reported to have poorer resolution and less sensitivity than MRI in patients with ONFH. ${ }^{15-18}$ It remains unclear whether BS can provide adequate screening of multiple osteonecrosis, except ONFH. The purpose of our study was to investigate the diagnostic power of increased bone uptake of technetium-99m labelled methylene diphosphonate ( ${ }^{99 \mathrm{~m}} \mathrm{Tc}-$ $\mathrm{MDP}$ ) for screening of $\mathrm{ONK}$ in comparison with MRI.

\section{Patients and methods}

Between August 1992 and August 1999125 patients were diagnosed as having $\mathrm{ONFH}$. Ninety four patients had undergone corticosteroid treatment (steroid related osteonecrosis) and 21 patients had a history of alcohol abuse (alcohol related osteonecrosis). Ten patients had no identifiable aetiological factors for osteonecrosis (idiopathic osteonecrosis). Three patients with alcohol related and four with idiopathic osteonecrosis did not undergo examination of the knee by BS or MRI at the time of ONFH diagnosis. Plain radiographs of 11 patients with steroid related osteonecrosis showed collapse of the joint surface or osteoarthritic changes of the tibiofemoral joints, including osteophytes and joint space narrowing. These 18 patients were excluded from our study. Thus a total of 214 knee joints in 107 patients with ONFH ( 51 female, 56 male) were examined by both BS and MRI, even when no knee symptoms were seen. The average age at diagnosis of ONFH was 42 years (range 17-79). The most common underlying disease in steroid related osteonecrosis was systemic lupus erythematosus in 26 patients.

The clinical status of the knee at diagnosis of $\mathrm{ONFH}$ was evaluated using the Hospital for Special Surgery score (HSS score). ${ }^{19}$ All patients were examined with anteroposterior and lateral radiographs of the knee. The stage of ONFC and ONTP was assessed according to the modified international classification by the Association of Research Circulation Osseous (ARCO) (table 1). ${ }^{20}$ Stage 1 diseases are not apparent on plain radiographs but are detectable with MRI. Stage 2 diseases are apparent on radiographs without collapse. Stage 3 diseases manifest collapse (collapsed stage). Stage 4 diseases show flattening of the femoral condyle or tibial plateau with joint space narrowing (osteoarthritic stage). In our study a two stage classification was used including stage 1 and 2 after excluding patients with stage 3 or stage 4 disease. The stage of the tibiofemoral joint was judged according to the stage of the more damaged medial or lateral compartment of the knee.

Patients were imaged using a 1.0 Tesla superconducting magnet (Magnetom Impact; Siemens, Erlangen, Germany) until 1997, and a 1.0 Tesla superconducting magnet (Signa, General Electric, Milwaukee, Wisconsin) after 1997. Before 1997 T1 weighted, spin echo images (T1WI) were obtained with repetition times (TR) of $500-800 \mathrm{~ms}$ and echo times (TE) of 15-43 ms, and T2 weighted spin echo images (T2WI, TR/TE $=2000-4500 / 96-117$ ms) were obtained with $5-10 \mathrm{~mm}$ thickness in the coronal and sagittal planes. After 1997 cross sectional images on the coronal plane were obtained with TORSO surface coil. All sequences used a $1.5 \mathrm{~mm}$ slice thickness with no interslice gap, a field view of $320 \mathrm{~mm}$, and one acquisition. T2WI (TR/TE $=3600 / 105$ ms), spoiled gradient recalled echo pulse sequences (SPGR, TR/TE $=14 / 2.3 \mathrm{~ms}$ ), and fat suppression SPGR (FS-SPGR) were ob-

Table 1 Modified ARCO (Association of Research Circulation Osseous) international staging system of osteonecrosis of the knee

Stage 0: All diagnostic studies normal; diagnosis by histology only

Stage 1: Plain radiographs, computed tomography normal; magnetic resonance imaging scintigraphy positive; biopsy positive Stage 2: $\quad$ Radiographs positive, but no collapse

Stage 3: Early flattening of dome; computed tomography or tomographs may be needed; further characterisation by amount of depression (in $\mathrm{mm}$ )

Stage 4: Flattening of femoral condyle or tibial plateau with joint space narrowing; possibly other signs of early osteoarthritis 


\begin{tabular}{|c|c|c|c|}
\cline { 3 - 4 } \multicolumn{2}{c|}{} & \multicolumn{2}{c|}{ Bone scintigraphy } \\
\cline { 3 - 4 } \multicolumn{2}{c|}{} & Positive & Negative \\
\hline MRI & Positive & a & b \\
\hline & Negative & c & d \\
\hline
\end{tabular}

Sensitivity $=a /(a+b)$

Specificity $=d /(c+d)$

Accuracy $=(a+d) /(a+b+c+d)$

Figure 2 Diagram showing the sensitivity, specificity, and accuracy of the bone scintigraphy (BS). a: true positive; $b$ : false negative; c: false positive; $d$ : true negative.

tained. The fat saturation pulse amplitude and frequency were optimised during the preimaging procedure. The image matrix was $256 \times$ 256 elements. SPGR and FS-SPGR image data on the coronal plane were resliced to evaluate on the mid-sagittal plane on a Macintosh computer using NIH image version 1.61/ ppc. The knee was divided into seven areas: the medial and lateral femoral condyles, distal femoral metaphysis, medial and lateral tibial plateau, proximal tibial metaphysis, and patella (fig 1). Necrotic lesions affecting subchondral bone beneath the joint surface were classified as either ONFC or ONTP, whereas lesions not affecting joint surface area were classified as either ONFM or ONTM. Normal fat intensity area surrounded by a low intensity band or diffuse low intensity area in each site on T1WI or SPGR was defined as osteonecrosis. ${ }^{8-10}$ MRI diagnosis was used as the gold standard in this study, because it was difficult to obtain histological diagnosis in multiple sites of asymptomatic cases. MR images were reviewed by one of the authors without benefit of clinical or radiological data.

The size of ONFC lesions on the midcoronal images of the femoral condyle, and anteroposterior (AP) location of ONFC lesions on the mid-sagittal images of the femoral condyle on T1WI or SPGR were evaluated according to a previous report. ${ }^{10}$ Briefly, the size of the lesion on the mid-coronal images was classified into three categories: small (S) affecting less than one third of the condyle, medium (M) - affecting more than one third and less than two thirds, and large (L)affecting more than two thirds. To evaluate the AP location, the medial or lateral condyle was divided into three zones on the mid-sagittal plane: anterior (A), middle (M), and posterior (P). The size and AP location of the lesions in the knee were judged using the more damaged

Table 2 Osteonecrosis of the knee

\begin{tabular}{lc}
\hline Site & $\begin{array}{c}\text { Number of knee } \\
\text { joints (\%) }\end{array}$ \\
\hline Total & $103(48)$ \\
Femoral condyle (ONFC) & $86(40)$ \\
$\quad$ Lateral & $82(38)$ \\
$\quad$ Medial & $52(24)$ \\
Distal femoral metaphysis (ONFM) & $32(15)$ \\
Tibial plateau (ONTP) & $2(0.9)$ \\
$\quad$ Medial & $2(0.9)$ \\
Lateral & $1(0.5)$ \\
Proximal tibial metaphysis (ONTM) & $21(10)$ \\
Patella (ONP) & $6(3)$ \\
\hline
\end{tabular}

medial or lateral compartment of the knee. A previous study ${ }^{10}$ indicated that when the lesion was large (L) or medium (M) and occupied more than two thirds of the AP location $(\mathrm{A}+\mathrm{M}+\mathrm{P}, \mathrm{A}+\mathrm{M}, \mathrm{M}+\mathrm{P}, \mathrm{A}+\mathrm{P})$, the knee showed a high collapse rate (11/25 condyles, $44 \%)$ and progressed to stage 3 or stage 4 disease in a mean of seven years (range 3-16). Thus the knees with the necrotic lesions which were L or $M$ size, and occupied more than two thirds of the AP location, were classified into the high risk group. On the other hand, when the lesion was medium $(\mathrm{M})$ and occupied less than one third of the AP location (A, M, P) or small (S), the knee did not collapse $(0 / 48$ condyles, $0 \%)$ or progress to stage 3 or stage 4 disease. Thus the knees with the necrotic lesions which were $M$ size and occupied less than one third of the AP location, or S size, were classified into the low risk group.

BS was performed after intravenous injection of $555 \mathrm{MBq}$ of ${ }^{99 \mathrm{~m}} \mathrm{Tc}-\mathrm{MDP}$. Three hours later, anterior and posterior view bone scintigrams of total body and bilateral knees were obtained using a gammacamera equipped with a high resolution, parallel hole collimator (gammavier-1 RC2600I, Hitachi, Tokyo, Japan). Bone scintigrams were reviewed by two of the authors who were unaware of the MRI data, without the benefit of clinical and radiological data. A consensus was then reached. Each of the seven areas of the knee described in the MRI method was evaluated. Increased bone uptake in each area was judged as positive on BS..$^{13}$ We calculated sensitivity, specificity, and accuracy of BS for ONK detection in comparison with MRI (fig 2). Mann-Whitney U test or Fisher's probability test was used for statistical analysis, and a $\mathrm{p}$ value less than 0.01 was considered significant.

\section{Results}

On MRI, ONK was detected in 103 knees of 62 patients (48\%). Ninety one knees in 54 patients with steroid related osteonecrosis and 12 knees in eight patients with alcohol related osteonecrosis had ONK. None of the patients with idiopathic osteonecrosis had ONK. Table 2 shows each site of ONK. ONFC was detected on MRI in 86 knees $(40 \%)-72$ knees in stage 1 and 14 in stage 2 . Two knees had stage 1 ONTP and both showed ONFC. The mean pain HSS score and total HSS score of these 62 patients with ONK was 27 (range 10-30) and 96 (range 65-100) points, respectively. Thirty eight knees in 23 patients showed clinical symptoms, including pain while climbing stairs (34 knees), and pain during walking (four knees). Although ONFM, ONTM, or ONP without ONFC affected 17 knees in 12 patients, only one knee manifested mild pain while climbing stairs.

Table 3 shows the sensitivity, specificity, and accuracy of BS for ONK detection. All ONK showed localised focal increased bone uptake on BS without linear pattern. Of 86 knees with positive findings of ONFC on MRI, 54 knees showed focal increased bone uptake on BS (true positive) (fig 3) and the other 32 showed negative findings (false negative). Sensitivity, 
Table 3 Bone scintigraphy and magnetic resonance imaging for detection of osteonecrosis of the knee

\begin{tabular}{|c|c|c|c|c|c|c|c|}
\hline & $\begin{array}{l}\text { True positive } \\
\text { (knees) }\end{array}$ & $\begin{array}{l}\text { False negative } \\
\text { (knees) }\end{array}$ & $\begin{array}{l}\text { False positive } \\
\text { (knees) }\end{array}$ & $\begin{array}{l}\text { True negative } \\
\text { (knees) }\end{array}$ & Sensitivity (\%) & Specificity (\%) & Accuracy (\%) \\
\hline Femoral condyles (ONFC) & 54 & 32 & 37 & 91 & 63 & 71 & 68 \\
\hline Distal femoral metaphysis (ONFM) & 1 & 31 & 4 & 178 & 3 & 98 & 84 \\
\hline Tibial plateau (ONTP) & 2 & 0 & 41 & 171 & 100 & 81 & 81 \\
\hline Proximal tibial metaphysis (ONTM) & 0 & 21 & 2 & 191 & 0 & 99 & 89 \\
\hline Patella (ONP) & 0 & 6 & 5 & 203 & 0 & 98 & 95 \\
\hline
\end{tabular}
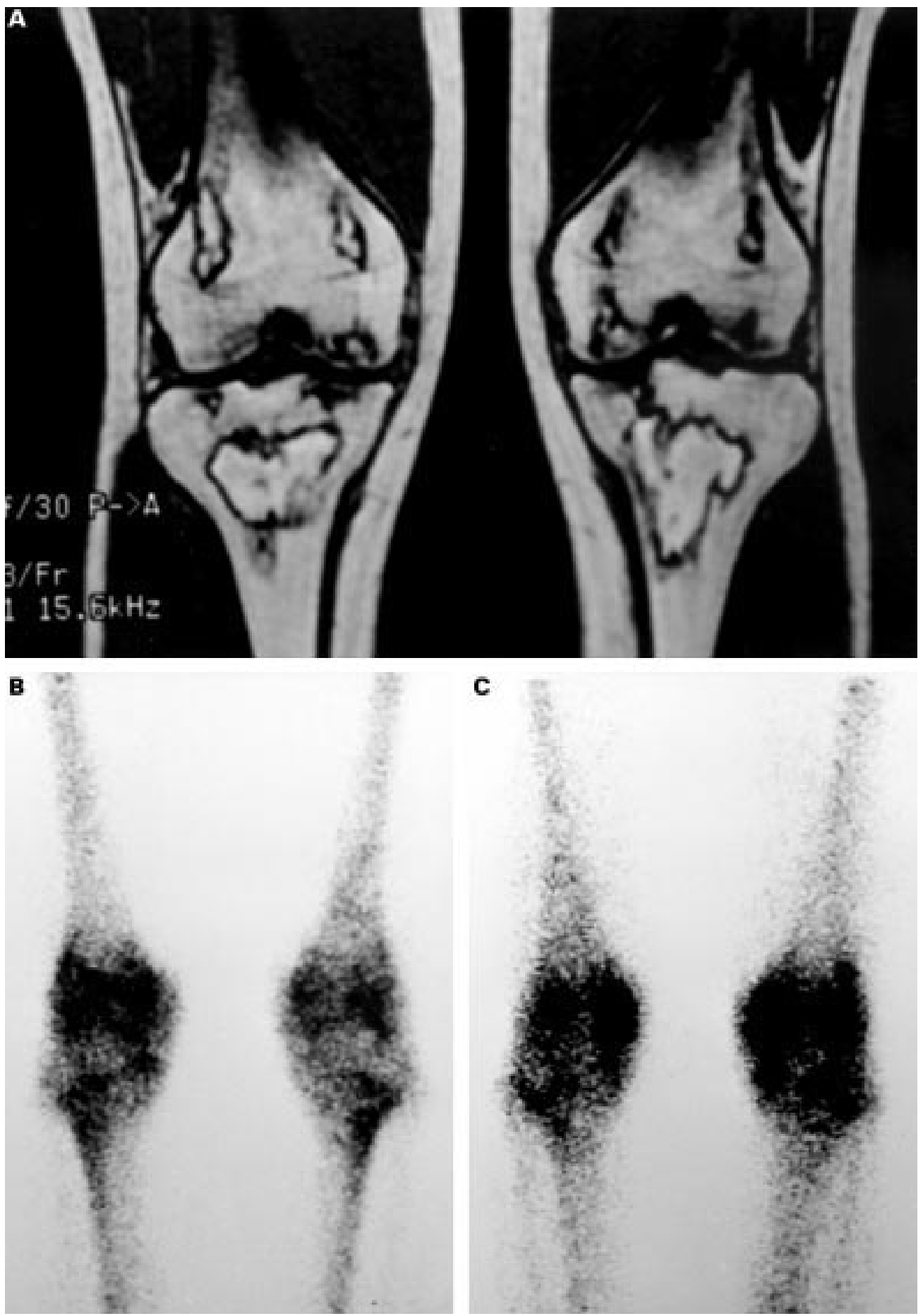

Figure 3 (A) A coronal MR image on spoiled gradient recalled echo pulse sequences ( $S P G R$, TR/TE $=14 / 2.3 \mathrm{~ms}$ ) of bilateral knees in a 21 year old woman who underwent bone marrow transplantation for acute lymphatic leukaemia and steroid treatment, showing osteonecrosis in bilateral femoral condyles (ONFC), distal femoral metaphysis (ONFM), tibial plateau (ONTP), and proximal tibial metaphysis (ONTM). (B) Anteroposterior and (C) posteroanterior bone scintigrams of bilateral knees showing focal increased bone uptake in bilateral femoral condyles, distal femoral metaphysis, tibial plateau, and proximal tibial metaphysis. 
Table 4 Patients with osteonecrosis of the femoral condyle (ONFC)

\begin{tabular}{|c|c|c|c|}
\hline & $\begin{array}{l}\text { True positive knees } \\
\text { (BS } \neq \text { positive/MRI } \\
\text { positive) }\end{array}$ & $\begin{array}{l}\text { False negative knees } \\
\text { (BS negative/MRI } \\
\text { positive) }\end{array}$ & p Value \\
\hline Total number of knee joints & 54 & 32 & \\
\hline Mean (SD) age at diagnosis of ONFH $\ddagger$ & $37(12)(17-58)$ & $35(13)(17-61)$ & $0.284^{\star}$ \\
\hline Sex $(M: F)$ & $25: 29$ & $14: 18$ & $0.827 \dagger$ \\
\hline \multicolumn{4}{|l|}{ Clinical status (mean (SD)) } \\
\hline HSS $\ddagger$ pain score & $26(5)(15-30)$ & $28(6)(10-30)$ & $0.137^{\star}$ \\
\hline HSS total score & $95(8)(65-100)$ & $97(5)(80-100)$ & $0.179^{\star}$ \\
\hline \multicolumn{4}{|l|}{ Radiological stage } \\
\hline 1 & 42 & 30 & $0.218^{\star}$ \\
\hline 2 & 12 & 2 & \\
\hline \multicolumn{4}{|l|}{ Size/anteroposterior location on MRI } \\
\hline High risk group & 34 & 4 & $<0.0001 \dagger$ \\
\hline $\mathrm{L} / \mathrm{A}+\mathrm{M}+\mathrm{P}$ & 9 & 0 & \\
\hline $\mathrm{L} / \mathrm{M}+\mathrm{P}$ & 3 & 1 & \\
\hline $\mathrm{M} / \mathrm{A}+\mathrm{M}+\mathrm{P}$ & 10 & 1 & \\
\hline $\mathrm{M} / \mathrm{M}+\mathrm{P}$ & 11 & 2 & \\
\hline $\mathrm{M} / \mathrm{A}+\mathrm{M}$ & 1 & 0 & \\
\hline Low risk group & 20 & 28 & \\
\hline $\mathrm{M} / \mathrm{M}$ & 5 & 2 & \\
\hline $\mathrm{S} / \mathrm{M}+\mathrm{P}$ & 2 & 1 & \\
\hline $\mathrm{S} / \mathrm{A}$ & 3 & 2 & \\
\hline $\mathrm{S} / \mathrm{M}$ & 2 & 1 & \\
\hline $\mathrm{S} / \mathrm{P}$ & 8 & 22 & \\
\hline
\end{tabular}

^Mann-Whitney U test.

$\dagger$ Fisher's exact probability test.

$\ddagger \mathrm{BS}=$ bone scintigraphy; HSS = Hospital for Special Surgery; MRI = magnetic resonance imaging; $\mathrm{ONFH}=$ osteonecrosis of the femoral head.

TThe size of the lesion on the mid-coronal images of the femoral condyle was classified into three categories: small $(\mathrm{S})$ : affecting less than one third of the condyle; medium $(\mathrm{M})$ : affecting more than one third and less than two thirds; and large (L): affecting more than two thirds. The anteroposterior location of the lesion on the mid-sagittal images of the femoral condyle was divided into three zones: anterior $(\mathrm{A})$, middle $(\mathrm{M})$, and posterior $(\mathrm{P})$.

specificity, and accuracy of BS for ONFC detection were $63 \%, 71 \%$, and $68 \%$, respectively. Between the 54 true positive and 32 false negative ONFC knees, there were no statistical differences in age at diagnosis of ONFH, sex, HSS score, or stage (table 4). However, we observed significant differences between the high risk and the low risk groups on MRI $(\mathrm{p}<0.0001)$. On BS, ONFC was detected in 34 of $38(89 \%)$ knees in the high risk group, and in 20 of $48(42 \%)$ knees in the low risk group. On the other hand, there were no statistical differences in age at diagnosis of $\mathrm{ONFH}$, sex, or existence of other knee necrotic lesions on MRI between 37 false positive and 91 true negative knees (table 5).

Sensitivity of BS for ONFM, ONTM, and ONP detection was $3 \%, 0 \%$, and $0 \%$, respectively (table 2). BS showed focal increased bone uptake in the whole region of the patella in five knees, but they were not identified as ONP by MRI (false positive) (fig 4). Sensitivity, specificity, and accuracy of BS for ONTP detection was $100 \%, 81 \%$, and $81 \%$, respectively.

\section{Discussion}

We defined increased bone uptake area as a positive indicator on BS. ${ }^{13}{ }^{14}$ All ONK lesions showed focal increased bone uptake and did not show "cold in hot pattern", which was specific to ONFH. ${ }^{21}$ The increased bone uptake reflects continuing bone reaction. In the femoral head of patients with ONFH, the blood supply is absent from the avascular segment and the area appears cold, whereas the reparative area with new vascular formation around the necrotic lesion appears hot on $\mathrm{BS} .{ }^{22}$ On the other hand, in the distal femur and the proximal tibia, anteroposterior bone volume is larger and blood supply by vascular anastomo$\operatorname{sis}^{5}$ is richer than in the femoral head. Thus bone uptake on the anteroposterior or posteroanterior plane tends to increase..$^{23}$ Therefore, all ONK lesions were thought to demonstrate focal bone uptake. The patterns of increased bone uptake looked partially like the patterns in osteoarthritis of the knee. ${ }^{24}{ }^{25}$ McCrae et al reported that late phase isotope retention occurred on the joint line (the tramline pattern), in the subchondral bone (extended pattern), throughout the joint (generalised), or was isolated to the patella (hot patella) in osteoarthritis of the knee. ${ }^{24}$ Boegard et al modified these four patterns of localised radionuclide uptake and identified three distinct patterns: point-like, tramline, and extended. ${ }^{25}$ In our study, localised focal increased bone uptake pattern was point-like or extended in all ONK patterns (fig 3), though no osteoarthritic changes were seen on plain radiographs. We did not find the linear pattern (tramline) in ONK patients.

Clinically, ONFC, ONTP, and ONP are more serious than ONFM or ONTM because subchondral bone is affected and these conditions may lead to collapse of the articular surface and degeneration of the tibiofemoral or patellofemoral joints. We found that ONFC was the most common site in ONK (40\%), which agreed with the findings of Shimizu et al (Steroid-induced multiple bone necroses: an analysis of 2000 joints in 250 patients. Paper presented at the Annual Meeting of the American Academy of Orthopaedic Surgeons, Anaheim, California, February, 1999). ONTP was rare $(0.9 \%)$ and was accompanied by ONFC. Among patients with ONFC, those with medium or large sized necrotic lesions on the coronal MRI views, which included two thirds or the entire surface area of the femoral condyle on the sagittal plane, have a high risk of subsequent collapse (the high risk group). ${ }^{10}$ Sensitivity of BS for detection of ONFC in the high risk group was $89 \%$. Therefore, BS is thought to be an adequate ONFC screening method.

Table 5 Patients without osteonecrosis of the femoral condyle (ONFC)

\begin{tabular}{llll}
\hline & $\begin{array}{l}\text { False positive knees (BS } \neq \\
\text { positive/MRI¥ negative) }\end{array}$ & $\begin{array}{l}\text { True negative knees (BS } \\
\text { negative/MRI negative) }\end{array}$ & $p$ Value \\
\hline Total number of knee joints & 37 & 91 & $0.819^{\star}$ \\
Mean (SD) age at diagnosis of ONFH $\neq$ & $45(14)(21-79)$ & $46(14)(20-79)$ & $0.899 \dagger$ \\
Sex (M:F) & $22: 15$ & $53: 38$ & $0.073 \dagger$ \\
Other necrotic lesions of the knee on MRI- & 8 & 8 & \\
\hline
\end{tabular}

^Mann-Whitney U test.

†Fisher's exact probability test.

$\ddagger \mathrm{BS}=$ bone scintigraphy; $\mathrm{MRI}=$ magnetic resonance imaging; $\mathrm{ONFH}=$ osteonecrosis of the femoral head.

$\uparrow$ Other necrotic lesions of the knee include the distal femoral metaphysis, proximal tibial metaphysis, tibial plateau, and patella. 
However, specificity of BS for detection of all ONFC was $71 \%$. To eliminate misdiagnosis of osteoarthritis of the knee on MRI, patients with osteoarthritic changes of the knee on plain radiographs were excluded from this study. Nevertheless, patients with preradiographical osteoarthritis might have been included in the 37 false positive patients, as Hutton et al reported in radiographically normal hands. ${ }^{26} 27$ Besides, it may be impossible to differentiate ONK completely from osteoarthritis of the knee using only BS without plain radiographs of the knee, because ONK and osteoarthritis of the knee have a similar appearance on BS- namely, point-like or extended pattern, ${ }^{24}{ }^{25}$ as mentioned above. Therefore, for the patients with increased bone uptake in the screening, it is desirable that $\mathrm{ONK}$ is diagnosed on $\mathrm{BS}$ and plain radiographs of the knee.

Sensitivity of BS for ONP detection was $0 \%$. Because only anterior and posterior views of BS were performed, the patellar region could not be clearly distinguished from the distal femoral region (the femoral condyles and metaphysis) despite marked localised focal increased bone uptake pattern. Although we could not use lateral projections in all patients because of machine limitations and patients'

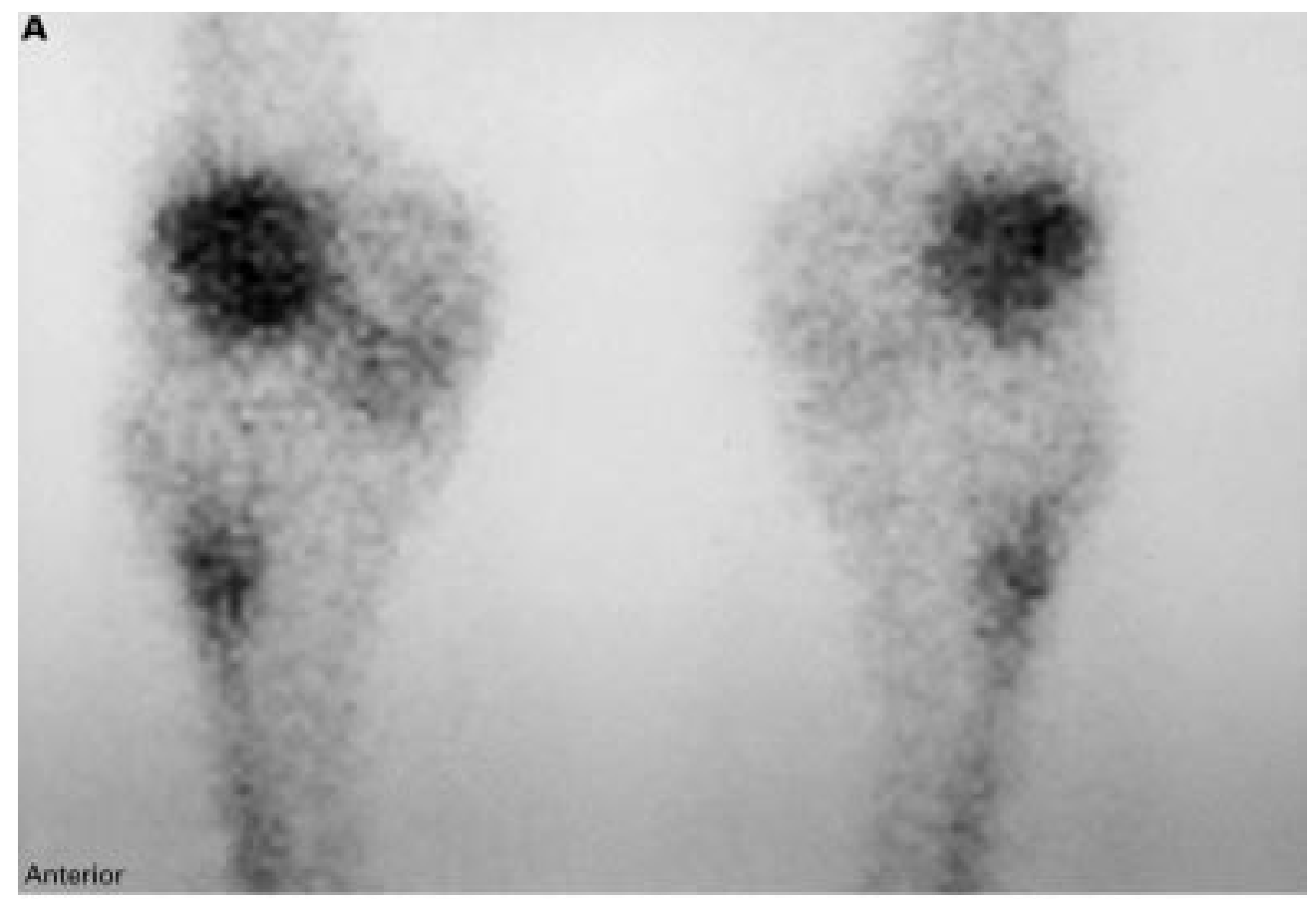

B

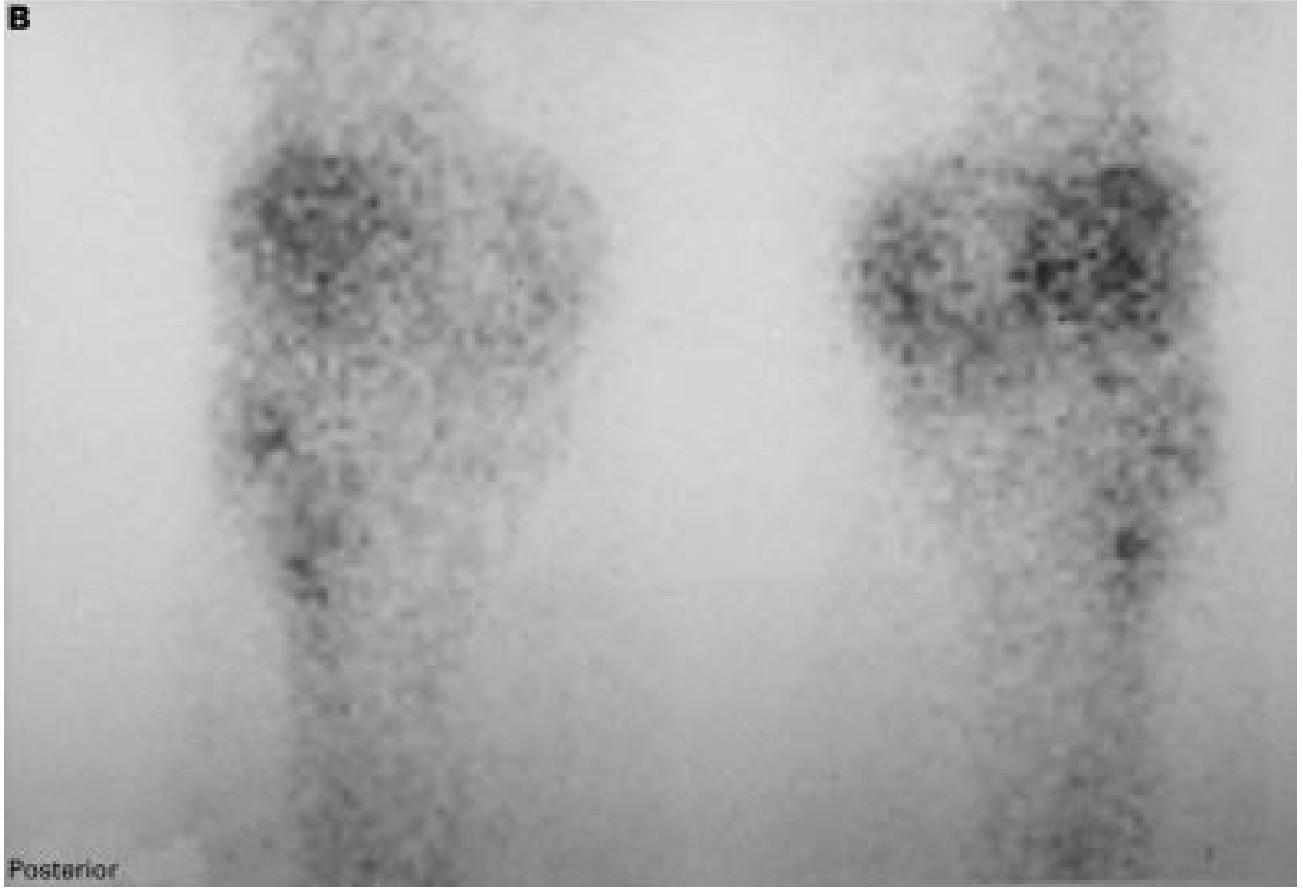

Figure 4 (A) Anteroposterior and (B) posteroanterior bone scintigrams of bilateral knees in a 61 year old man with alcohol abuse, showing increased bone uptake in the whole area of the patella (hot patella pattern). Bilateral knees showed no osteoarthritic changes radiologically and no osteonecrosis on MRI. 
conditions, these projections of $\mathrm{BS}^{26}$ could distinguish the anteroposterior location of the necrotic lesions and would increase the information provided by BS. It might improve not only the sensitivity but also the specificity and the accuracy. The "hot patella pattern" ${ }^{24}$ was seen in the entire patellar region of five knees (fig 4), though normal conditions were indicated on MRI (false positive). Boegard et al also reported that in the patella, six of seven observations of increased bone uptake showing the hot patella pattern did not correspond to subchondral lesions in osteoarthritis. ${ }^{25}$ This pattern in the patella may be indicative of normal conditions.

We found low BS sensitivity for detection of ONFM $(3 \%)$ and ONTM $(0 \%)$, which reflects the fact that continuing bone reaction in the metaphysis is poorer and smaller than that at the subchondral bone. In accordance with these findings, there were no clinical symptoms in the patients with ONFM or ONTM alone, except in one knee, and those necrotic sites have no possibility of knee collapse. We do not believe that these findings mean that $\mathrm{BS}$ is inadequate for ONK screening.

In conclusion, our results indicate that BS is useful for screening large ONK in patients with non-traumatic ONFH because $89 \%$ of patients with ONFC who had a high risk of collapse of the knee were detected by BS. Although osteonecrosis of the shoulder, the ankle, and the other sites are less common than ONK, ${ }^{12}$ BS may be useful for screening multiple osteonecrosis because many joints can be examined at one time on total body images.

This work was supported in part by a grant from the Japanese Investigation Committee under the auspices of the Ministry of Health and Welfare, Japan Society for the Promotion of Science, and Hip Joint Foundation of Japan, Inc. No benefits in any form have been received or will be received from a commercial party related directly or indirectly to the subject of this article.

1 LaPorte DM, Mont MA, Mohan V, Jones LC, Hungerford DS. Multifocal osteonecrosis. J Rheumatol 1998;25:196874.

2 Mont MA, Jones LC, LaPorte DM (Collaborative Osteonecrosis Group). Symptomatic multifocal osteonecrosis. A multicenter study. Clin Orthop 1999;369:312-26.

3 Mankin HJ. Nontraumatic necrosis of bone (osteonecrosis). N Engl J Med 1992;326:1473-9.

4 Jones JP. Intravascular coagulation and osteonecrosis. Clin Jones JP. Intravascular coa

5 Shim SS, Leung BA. Blood supply of the knee joint. A microangiographic study in children and adults. Clin microangiographic study
Orthop 1986;208:119-25.

6 Motohashi M, Morii T, Koshino T. Clinical course and roentgenographic changes of osteonecrosis in the femoral condyle under conservative treatment. Clin Orthop 1991 266:156-61.
7 Sasaki T, Yagi T, Monji J, Yasuda K, Masuda T Steroid-induced osteonecrosis of the femoral condyle: a clinical study of eighteen knees in ten patients. Journal of the Japanese Orthopedic Association 1986;60:361-72.

8 Healy W. Osteonecrosis of the knee detected only by magnetic resonance imaging: case reports. Orthopedics 1991;14:703-4.

9 Pollack MS, Dalinka MK, Kressel HY, Lotke PA, Spritzer CE. Magnetic resonance imaging in the evaluation of suspected osteonecrosis of the knee. Skeletal Radiol 1987;16: pected os

10 Sakai T, Sugano N, Ohzono K, Matsui M, Hiroshima K, Ochi T. MRI evaluation of steroid- or alcohol-related osteonecrosis of the femoral condyle. Acta Orthop Scand 1998;69:598-602.

11 Mont MA, Tomek IM, Hungerford DS. Core decompression for avascular necrosis of the distal femur. Long term followup. Clin Orthop 1997;334:124-30.

12 Mont MA, Myers TH, Krackow KA, Hungerford DS. Total knee arthroplasty for corticosteroid associated avascular necrosis of the knee. Clin Orthop 1997;338:124-30.

13 Burt RW, Matthews TJ. Aseptic necrosis of the knee: bone scintigraphy. AJR 1982;138:571-73.

14 Minoves M, Riera E, Constansa JM, Bassa P, Setoain J, Domenech FM. Multiple aseptic bone necrosis detected by Tc-99m MDP bone scintigraphy in a patient with systemic lupus erythematosus on corticosteroid therapy. Clin Nucl Med 1998;23:48-9.

15 Totty WG, Murphy WA, Ganz WI, Kurmar B, Daum WJ, Siegel BA. Magnetic resonance imaging of the normal and ischemic femoral head. AJR 1984;143:1273-80.

16 Mitchell DG, Rao VM, Dalinka MK, Spritzer CE, Alavi A, Steinberg ME, et al. Femoral head avascular necrosis: correlation of MR imaging, radiographic staging, radionuclide imaging, and clinical findings. Radiology 1987;162:70915.

17 Beltran J, Herman LJ, Burk JM, Zuelzer WA, Clark RN, Lucas JG, et al. Femoral head avascular necrosis: MR imaging with clinical-pathologic and radionuclide correlation. Radiology 1988;166:215-20.

18 Fordyce MJF, Solomon L. Early detection of avascular necrosis of the femoral head by MRI. J Bone Joint Surg Br 1993;75:365-7.

19 Ranawat CS, Insall J, Shine J. Duo-condylar knee arthroplasty: hospital for special surgery design. Clin Orthop 1976;120:76-82.

20 Stulberg BN. Editorial comment. Clin Orthop 1997;334: $2-5$.

21 Sugano T, Kubo T, Takaoka K, Ohzono K, Hotokebuchi T, Matsumoto $\mathrm{T}$, et al. Diagnostic criteria for non-traumatic osteonecrosis of the femoral head. A multicentre study. J Bone Joint Surg Br 1999;81:590-5.

22 D'Ambrosia R, Shouji H, Riggins RS, Stadalnik RC, DeNardo GL. Scintigraphy in the diagnosis of osteonecrosis. Clin Orthop 1978;130:139-43.

23 Nishioka J. The diagnostic value of bone scintiscanning in aseptic osteonecrosis of the femur. Comparative study between bone scintigram and histological findings. Journal of the Japanese Orthopedic Association 1979;53:429-40 (in Japanese)

24 McCrae F, Shouls J, Dieppe P, Watt I. Scintigraphic assessment of osteoarthritis of the knee joint. Ann Rheum Dis 1992;51:938-42.

25 Boegard T, Rudling O, Dahlstrom J, Dirksen H, Petersson $\mathrm{IF}$, Jonsson $\mathrm{K}$. Bone scintigraphy in chronic knee pain: comparison with magnetic resonance imaging. Ann Rheum Dis 1999;58:20-6.

26 Hutton CW, Higgs ER, Jackson PC, Watt I, Dieppe PA. ${ }^{99 \mathrm{~m}} \mathrm{Tc}$ HMDP bone scanning in generalised arthritis. I. Comparison of the standard radiograph and four hour bone scan image of the hand. Ann Rheum Dis 1986;45: 617-21.

27 Hutton CW, Higgs ER, Jackson PC, Watt I, Dieppe PA. ${ }^{9 \mathrm{~m}} \mathrm{Tc}$ HMDP bone scanning in generalised arthritis. II. The four hour bone scan image predicts radiographic change. Ann Rheum Dis 1986;45:622-6. 